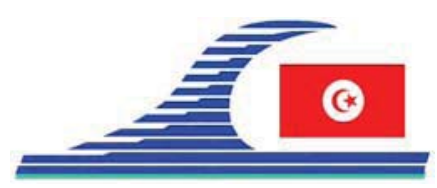

Conférence Méditerranéenne Côtière et Maritime EDITION 1, HAMMAMET, TUNISIE (2009)

Coastal and Maritime Mediterranean Conference

Disponible en ligne - http://www.paralia.fr - Available online

\title{
AMPHISOLAR. \\ Les salines, entre eau et énergie, de nouvelles « Routes du sel» pour l'Union de la Méditerranée
}

\author{
Jean-Louis PACITTO ${ }^{1}$, Odile JACQUEMIN ${ }^{2}$
}

1. Architecte urbaniste, Chercheur prospectiviste.

2. Architecte urbaniste, Docteur en Histoire des territoires.

Membres du Pôle Mer PACA et du Réseau Français de Recherche Côtière (RFRC), Groupement d'Intérêt Scientifique AMPHIBIA Aquapolis / MALTAE,

32 Chemin St Lazare, 83400 Hyères, France.

jlpacitto@gmail.com ; odilejacquemin@gmail.com

\section{Résumé :}

AMPHISOLAR désigne un concept d'étangs et de bassins solaires à gradient de salinité venant en appui de projets côtiers durables, lesquels consistent à revaloriser la multifonctionnalité de salines en activité ou en friches autour du bassin méditerranéen.

L'objet de cette contribution est de témoigner de développements cherchant à concilier durabilité environnementale et faisabilité économique au moyen de l'exploration de différentes possibilités de rénovation ou de reconversion de salines, avec une vision très intégrée de la valorisation de ces espaces côtiers méditerranéens. Cette question intéresse aussi la protection ou la défense et la réhabilitation d'espaces à même d'être affectés par le recul du trait côtier et soumis à une avancée du biseau salé, face au changement climatique. Devant la gravité des prédictions sur les effets de ce changement, il convient sans attendre de limiter tous azimuts les émissions de GES; et face aux risques de pénurie d'énergies fossiles et de ressources, y compris dans des secteurs comme l'alimentaire, il incombe aux acteurs de l'aménagement du territoire de se pencher simultanément sur ces problématiques d'intégration par une mise en synergie de divers processus adaptatifs et économes. Ainsi, dans les secteurs littoraux à forts enjeux telles les zones côtières périurbaines confrontant des espaces humides : salins, lagunes, deltas, zones intertidales, lacs et marais (eau douce, saumâtre ou salée), il s'agira d'innover avec la programmation de projets répondant tout autant aux critères de durabilité environnementale qu'à ceux de faisabilité économique.

On peut observer que de nombreux littoraux méditerranéens de type lagunaire salé ou non sont déjà ou seront particulièrement concernés par ces phénomènes (Exemples de coopération envisageables entre espaces significatifs des façades littorales en Méditerranée, tels Giraud ou Aigues-Mortes en France, Sfax en Tunisie, etc.., à mettre pertinemment en miroir), avec des évolutions plus ou moins différenciées; et dans ce sens la perspective de tels projets devrait avoir le mérite d'ouvrir un espace-temps de fortes coopérations en Méditerranée.

$$
\text { DOI: } 10.5150 / \mathrm{cmcm} .2009 .079-4
$$




\section{Mots-clés :}

Etang solaire à gradient de salinité - Biomasse - Biodiesel - Ethanol - Dessalement Production électrique - Aquaculture - Gaz de serre chaude - Changement climatique

\section{Eau et énergie : des processus naturels pour des productions renouvelables et un développement durable}

Description des dispositifs productifs de base : Un étang ou bassin solaire est un dispositif de collecte et de stockage naturels de l'énergie tirée du rayonnement solaire, avec une chaleur qui se trouve littéralement piégée dans la couche saturée en fond de dispositif. Un gradient de salinité maintenu avec les deux autres strates aqueuses, l'une dite intermédiaire (couche stable), l'autre supérieure (couche d'évaporation), en interdit les convections et échanges internes, évitant les pertes, ce qui permet à la saumure d'atteindre des températures relativement élevées $\left(60-80^{\circ} \mathrm{C}\right)$.

Conditions d'implantation : Un étang ou bassin solaire nécessite de disposer d'une large bande de terre (elle pourrait être stérile), de beaucoup de soleil (donc de temps clair et de vent), et la disponibilité de sel (tel le chlorure de sodium de l'eau de mer). Les étangs solaires utilisent généralement un à deux mètres de hauteur d'eau et des gradients de salinité permettant d'atteindre des températures élevées.

Productions écologiques et multiplicité d'usages: Stockée, une importante énergie thermique, utilisable indépendamment du temps et de la saison, peut être retirée de l'étang solaire sous forme de saumure chaude. L'énergie thermique est fournie sans combustible, réduisant la pollution; les autres ressources d'énergie sont économisées.

Cette chaleur solaire peut donc générer une électricité "verte" et de l'eau douce. La cogénération d'électricité et la désalinisation de l'eau sont d'ailleurs des principes bien acceptés dans de nombreux pays. Les étangs solaires couplés avec des unités de dessalement peuvent être utilisés pour purifier ou réduire la contamination d'eau minérale, et l'étang lui-même peut devenir le réceptacle des déchets produits.

Inconvénients et objectifs de maitrise des impacts : Etant donné la forte augmentation du nombre et de la capacité des installations de dessalement prévues au cours des années à venir le long des côtes, se pose avec acuité la question de l'élimination des effluents salins qui peuvent être des sources de pollutions (Projet de recherche DESALIN Institut Paul Ricard Les Embiez Var France, labellisé Pôle Mer PACA). De nouveaux modèles de traitement des déchets optimisant la récupération des métaux depuis les sous-produits de la production de saumure voient le jour. La meilleure façon d'atteindre cet objectif est sans doute d'adapter la recherche théorique traitant de la récupération des ressources du saumure au pilotage du process général, intégré à la production de l'eau potable, et idéalement, liée au principe de cogénération.

La réalisation à l'échelle territoriale de projets de développement intégrant ce domaine d'applications apparaîtrait comme le symbole de la prise de conscience écologique de l'industrie du dessalement, apportant une contribution positive (à ce qui est aujourd'hui 
un «surcoût») au problème de l'élimination des effluents salins. Un aperçu de certains travaux de recherche appliquée et d'expérimentations à petite échelle dans différents pays donne déjà une indication des avantages potentiels d'une telle approche. De nouveaux process ont été approfondis et montrent la voie (Integrated power, water and salt generation : Dr M. Ahmed, Dr A. Arakel, D.Hoey, M. Coleman).

Le défi du recyclage des résidus intégré à un process global de production salinière :

Cela consiste à lier le traitement des eaux salines à la production d'électricité, au dessalement, au développement d'une aquaculture (production de poissons et crevettes), à la concentration des saumures et à la récupération des ressources minérales, etc... le tout vu comme un véritable système intégré, de type écosystémique. Il y a là matière à $R$ et $\mathrm{D}$ ou $\mathrm{R}$ et I, s'agissant de formaliser in situ la façon de réaliser au mieux l'intégration des différents processus, d'en améliorer l'intelligence et l'efficacité globales. Ceci considéré, il nous est apparu que les salines en activité ou en friches tout autour du bassin méditerranéen, considérées comme structures "naturelles » de production, bien calées « entre terre et mer» et présentant des caractéristiques adaptées, étaient en mesure d'intégrer un certain nombre de dispositifs démonstrateurs (Salines de Giraud, salines d'Aigues-Mortes, salines de Sfax, ...), en support des 3 projets pilotes d'aménagement à mettre en miroir dans le cadre de l'Union de la Méditerranée.

\section{2. Étangs solaires et friches salinières : un élan et une vocation renforcée pour les territoires autour d'une production salinière « nouvelle génération ».}

Avantages de l'intégration de fonctions productives dans un processus d'aménagement durable des littoraux :

Le système solaire de production industrielle de chaleur d'un étang est innovant, efficace en termes de coût d'investissement, et permet la capture et le stockage d'énergie pour de multiples applications. Conjuguer à la commercialisation d'une énergie renouvelable, qui réduit la dépendance littorale envers les combustibles fossiles, à une nouvelle forme de prospérité économique, une vocation réaffirmée autour du sel, nécessite de concevoir des projets intégrés qui soient démonstratifs et de dimension régionale. AMPHISOLAR, consortium réunissant des partenaires des 2 rives de la Méditerranée (Universités, PMI/PME, Grands groupes, Sociétés salinières, Collectivités territoriales) est en mesure d'initier et de mener un projet de cette facture autour de 3 systèmes maillés d'étangs solaires, dans des contextes urbains littoraux différents. Adossés à des espaces saliniers tunisiens et français, dont l'eau des étangs, marais et bassins programmés in situ capture et stocke l'énergie solaire, des recherches seront menées pour en décliner les potentialités connexes de développement. Il s'agit d'intégrer la chaleur des étangs non seulement dans le processus courant de la production du sel et de ses produits dérivés, mais aussi pour l'aquaculture, pour l'alimentation et la santé. AMPHISOLAR estime que l'installation d'étangs solaires permettra la production du sel et de ses sous-produits à des coûts plus compétitifs, vu 
les ressources générées par tout un panel de produits dérivés. Cette amélioration de la rentabilité devrait stimuler les investissements, le chiffre d'affaires, les bénéfices, et impliquer de nouveaux métiers, donc des emplois. Au niveau local ce serait une nouvelle impulsion donnée aux zones industrielles salinières en difficulté ou en friches, avec un chômage dont on redoute l'importance, un patrimoine industriel et naturel en péril, et une économie locale déprimée, à fortiori en ce temps de crise. Des étangs solaires ont déjà été installés, avec au moins 60 systèmes qui ont été construits à travers le monde (Pyramid Hill, Northern-Victoria, Australia ; El Paso University, Texas, EU ; Ecole Nationale d'Ingénieurs de Tunis, Tunisie ; Université d'Ancona, Italie ; Centre d'études solaires, Tajoura, Lybie), principalement pour la fourniture de chaleur à l'industrie. Il n'y a pas eu encore en France et en Tunisie d'applications « commerciales » de l'énergie solaire des étangs, utilisable « en boucle » localement pour dynamiser l'activité en place et revaloriser des héritages séculaires ayant contribué à modeler bien des paysages littoraux et assuré leur pérennité.

En tant que concept novateur de développement, les espaces saliniers reconvertis autour de leurs étangs solaires, devenus à la fois ressources et outils, seront d'autant plus attractifs que le coût de la construction des bassins et de leur maintenance sera minorée vu l'augmentation attendue des coûts de l'énergie conventionnelle. Cette démultiplication de vocations pourra s'appliquer en Méditerranée pour mener des opérations cohérentes d'aménagement durable du littoral.

La maitrise des technologies nécessaires est parfaitement assurée compte tenu du profil et de l'expérience internationale des partenaires d'Amphisolar dans ces domaines.

Les étangs solaires offrent une énergie renouvelable qui réduit fortement les émissions de GES. Les étangs solaires peuvent apporter de nouvelles «terres fertiles» aux agriculteurs touchés par le biseau salé pour une autre utilisation productive.

\section{Bilans et perspectives}

Les étangs solaires peuvent produire de la chaleur $\left(60-80^{\circ} \mathrm{C}\right)$ pour une grande variété d'applications à un coût moyen équivalent aux 2/3 du coût du GPL. Celui-ci n'étant pas souvent disponible dans ces zones, les étangs solaires seront très rentables pour des applications de chauffage à basse température, (agriculture, élevage, ...). Les projections indiquent que le développement de la commercialisation de cette énergie permettrait de réduire les émissions de GES de plus de 10 mégatonnes par an et générerait de l'ordre de $2000 \mathrm{GWh}$ par an d'électricité verte. Au niveau international, les économies pourraient s'avérer très importantes et participer pour une grande part à la lutte contre les effets du réchauffement climatique et la raréfaction des ressources fossiles. De fait le concept AMPHISOLAR permet à toutes les collectivités littorales jouant la carte de l'innovation dans l'aménagement durable de leurs espaces saliniers de revendiquer et bénéficier pleinement de leur droit légitime d'expérimentation en ce domaine. 